\title{
A Closer Look At Feature Space Data Augmentation For Few-Shot Intent Classification
}

\author{
Varun Kumar, Hadrien Glaude, Cyprien de Lichy, Wlliam Campbell \\ Amazon Alexa \\ Cambridge, MA, USA \\ $\{$ kuvrun, hglaude, cllichy, cmpw\}@amazon.com
}

\begin{abstract}
New conversation topics and functionalities are constantly being added to conversational AI agents like Amazon Alexa and Apple Siri. As data collection and annotation is not scalable and is often costly, only a handful of examples for the new functionalities are available, which results in poor generalization performance. We formulate it as a Few-Shot Integration (FSI) problem where a few examples are used to introduce a new intent. In this paper, we study six feature space data augmentation methods to improve classification performance in FSI setting in combination with both supervised and unsupervised representation learning methods such as BERT. Through realistic experiments on two public conversational datasets, SNIPS, and the Facebook Dialog corpus, we show that data augmentation in feature space provides an effective way to improve intent classification performance in fewshot setting beyond traditional transfer learning approaches. In particular, we show that (a) upsampling in latent space is a competitive baseline for feature space augmentation (b) adding the difference between two examples to a new example is a simple yet effective data augmentation method.
\end{abstract}

\section{Introduction}

Virtual artificial assistants with natural language understanding (NLU) support a variety of functionalities. Throughout the lifespan of the deployed NLU systems, new functionalities with new categories, are regularly introduced. While techniques such as active learning (Peshterliev et al., 2018), semi-supervised learning (Cho et al., $2019 \mathrm{~b}$ ) are used to improve the performance of existing functionalities, performance for new functionalities suffers from the data scarcity problem.

Recently, Few-Shot Learning has been explored to address the problem of generalizing from a few examples per category. While it has been extensively studied (Koch et al., 2015; Snell et al., 2017; Vinyals et al., 2016) for image recognition, a little attention has been paid to improve NLU performance in the low-data regime. Moreover, researchers have been mostly working on the unrealistic setting that considers tasks with few categories unseen during (pre)training, each with only a few examples, and introduces new categories during test time. We argue that a more realistic setting is Few-Shot Integration (FSI) where new categories with limited training data are introduced into an existing system with mature categories. FSI is well aligned with the goal of lifelong learning of conversational agents and measures the performance in a real-life system setting when only a few examples of a new class are added to the existing data from the old classes. To address the poor generalization in data scare scenarios, several pre-training methods such as ELMo (Peters et al., 2018), Generative pre-trained Transformer (Radford et al., 2018), BERT (Devlin et al., 2018), have been proposed which are trained on a large amount of unannotated text data. Such pre-trained models can be fine-tuned on a particular NLP task and have shown to greatly improve generalization. However, in FSI setting where only a handful of examples are provided, building accurate NLU model is still a challenging task.

In this paper, we focus on Feature space Data Augmentation (FDA) methods to improve the classification performance of the categories with limited data. We study six widely different feature space data augmentation methods: 1) upsampling in the feature space UPSAMPLE, 2) random perturbation PERTURB, 3) extrapolation (Devries and Taylor, 2017) EXTRA, 4) conditional variational auto-encoder (CVAE) (Kingma and Welling, 2013) CVAE, 5) delta encoder that have been especially designed to work in the few-shot 
learning setting (Schwartz et al., 2018) DELTA, 6) linear delta which is a linear version of the delta encoder Linear. While Upsample, Perturb, EXTRA and LINEAR doesn't require any training beyond hyper-parameter optimization, DELTA and CVAE are trained deep neural network generators.

We compare these six FDA techniques on two open datasets for Intent Classification (IC) : SNIPS (Coucke et al., 2018) and Facebook Dialog corpus (Gupta et al., 2018). We show that BERT combined with LINEAR data augmentation provides an effective method to bootstrap accurate intent classifiers with limited training data. We make the following contributions:

1. We propose the FSI evaluation, a relaxation of the few-shot learning setting that aims to better model the requirement of modern NLU systems. We provide a comprehensive evaluation of FSI for text classification and show that UPSAMPLE and PERTURB are simple yet efficient baselines that are often neglected in few-shot learning evaluations.

2. We provide an in-depth analysis of various FDA methods. We show that complex methods such as DELTA and CVAE do not always improve over simple methods like LINEAR, and the performance heavily depends on the feature extractor.

3. Finally, we provide guidance on when and how to apply FDA for FSI. We show that FDA consistently provides gains on top of the unsupervised pre-training methods such as BERT in FSI setting.

\section{Related work}

Few-shot learning has been studied extensively in the computer vision domain. In particular, several metric learning based methods (Koch et al., 2015; Vinyals et al., 2016; Snell et al., 2017; Rippel et al., 2015) has been proposed for few-shot classification where a model first learns an embedding space and then a simple metric is used to classify instances of new categories via proximity to the few labeled training examples embedded in the same space. In addition to metric-learning, several meta-learning based approaches (Ravi and Larochelle, 2016; Li et al., 2017; Finn et al., 2017) have been proposed for few-shot classification on unseen classes.
Recently, Few-Shot Learning on text data has been explored using metric learning (Yu et al., 2018; Jiang et al., 2018). In (Yu et al., 2018), authors propose to learn a weighted combination of metrics obtained from meta-training tasks for a newly seen few-shot task. Similarly, in (Cheng et al., 2019), authors propose to use meta-metriclearning to learn task-specific metric that can handle imbalanced datasets.

Generative models are also widely used to improve classification performance by data augmentation. For example, generative models are used for data augmentation in image classification (Mehrotra and Dukkipati, 2017; Antoniou et al., 2018; Zhang et al., 2018), text classification (Gupta, 2019), anomaly detection (Lim et al., 2018). Data augmentation through deformation of an image has been known to be very effective for image recognition. More advanced approaches rely on Auto-Encoders (AEs) or Generative Adversarial Networks (GANs). For example, in (Mehrotra and Dukkipati, 2017) the authors combine metric-learning with data augmentation using GANs for few-shot learning. However, classical generative approaches require a significant amount of training data to be able to generate good enough examples that will improve classification accuracy. To overcome this challenge, (Hariharan and Girshick, 2017) proposed to augment the training data in the feature space. This both eases the generation problem and enforces generation of discriminative examples. In addition, the authors propose to transfer deformations from base classes to new classes, which allows circumventing the data scarcity problem for new classes. Finally, in (Schwartz et al., 2018), authors used an Autoencoder to encode transformations between pairs of examples of the same class and apply them to an example of the new class.

Generative models are a good candidate for FSI tasks, as one can just combine the generated data for new classes with the old classes training data (Hariharan and Girshick, 2017; Wang et al., 2018). For text classification, several text generation based data augmentation techniques have also been explored (Hou et al., 2018; Zhao et al., 2019; Guu et al., 2018; Yoo et al., 2018; Cho et al., 2019a). However, generating discrete sequences, e.g. text, is known to be quite difficult and requires lots of training data. That is why, in this paper, we focus on generative models, which augment data 


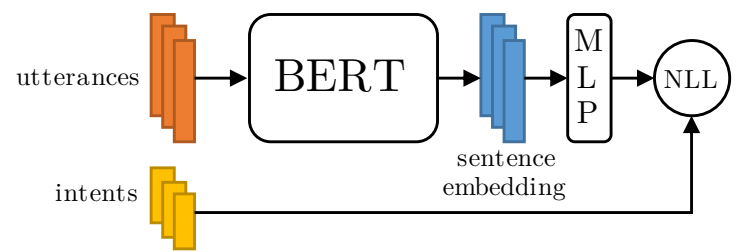

(a) Learning the feature space

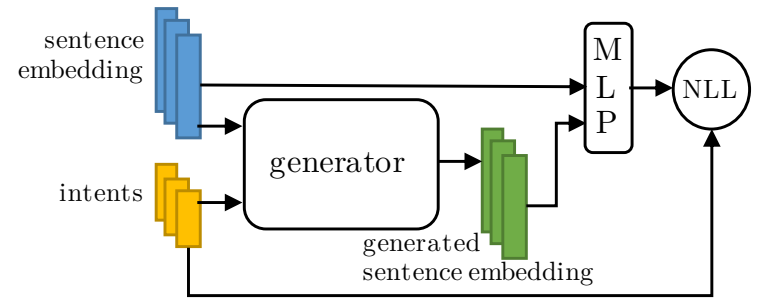

(b) Learning with augmented data

Figure 1: Data augmentation in the feature space

in latent(feature) space to solve a few-shot integration problem for text classification.

\section{Data Augmentation in Feature Space}

Feature space data Augmentation (FDA) is an effective method to improve classification performance on different ML tasks (Chawla et al., 2002; Hariharan and Girshick, 2017; Devries and Taylor, 2017; Guo et al., 2019). As shown on Figure 1, FDA techniques usually work by first learning a data representation or feature extractor, and then generating new data for the low resource class in the feature space. After generating data, a classifier is trained with real and augmented data.

For IC, we finetune a pre-trained English BERTBase uncased model ${ }^{1}$ to build our feature extractor. The BERT model has 12 layers, 768 hidden states, and 12 heads. We use the pooled representation of the hidden state of the first special token ([CLS]) as the sentence representation. A dropout probability of 0.1 is applied to the sentence representation before passing it to the 1-layer Softmax classifier. BERT Encoder and MLP classifier are fine-tuned using cross-entropy loss for IC task. Adam (Kingma and $\mathrm{Ba}, 2014$ ) is used for optimization with an initial learning rate of $5 e-5$.

For data augmentation, we apply six different FDA methods, described below, to generate new examples in the feature space. Finally, we train a 1- layer Softmax classifier as in the feature learning phase.

\footnotetext{
${ }^{1}$ https://github.com/huggingface/pytorch-transformers
}

\subsection{Upsampling}

The simplest method to augment training data for underrepresented categories is to duplicate the existing training data. Upsampling is a well studied technique to handle the class imbalance problem (Estabrooks et al., 2004). We show that for intents with limited labeled data, upsampling the existing data in latent space consistently improves model performance, and thus is a good baseline method for FDA techniques. We call this method UPSAMPLE.

\subsection{Random Perturbation}

Adding random noise to the existing training data is another simple yet effective data augmentation technique. Random perturbation data augmentation has been previously used to improve the performance of classification models as well as for sequence generation models. For example, (Kurata et al., 2016) applied additive and multiplicative perturbation to improve the text generation for data augmentation. In our experiments, we apply both additive and multiplicative perturbation to the existing training data. We sample noise from a uniform distribution $[-1.0,1.0]$. We use PERTURB to refer to this method.

\subsection{Conditional VAE}

Conditional Variational Autoencoder (CVAE) is an extension of Variational Autoencoder (VAE) (Kingma and Welling, 2013) which can be used to generate examples for a given category. All components of the model are conditioned on the category. First, we train CVAE on the sentence representations and then generate new examples by sampling from the latent distribution. The encoder and decoder sub-networks are implemented as multi-layer perceptrons with a single hidden layer of 2048 units, where each layer is followed by a hyperbolic tangent activation. The encoder output $Z$ is 128-dimensional. Mean Square Error (MSE) loss function is used for reconstruction. All models are trained with Adam optimizer with the learning rate set to $10-3$.

\subsection{Linear Delta}

A simple method to generate new examples is to first learn the difference between a pair of examples, and then add this difference to another example. In this case, we first compute the difference $X_{i}-X_{j}$ between two examples from the same 
class and then add it to a third example $X_{k}$ also from the same class as shown in (1). We use LiNEAR to refer to this method.

$$
\hat{X}=\left(X_{i}-X_{j}\right)+X_{k}
$$

\subsection{Extrapolation}

In (Devries and Taylor, 2017), authors proposed to use extrapolation to synthesize new examples for a given class. They demonstrated that extrapolating between samples in feature space can be used to augment datasets. In extrapolation, a new example, $\hat{X}$ is generated according to (2). In our experiments, we use $\lambda=0.5$. We call this method EXTRA.

$$
\hat{X}=\left(X_{i}-X_{j}\right) * \lambda+X_{i}
$$

\subsection{Delta-Encoder}

Delta-Encoder (Schwartz et al., 2018) extends the idea of learning differences between two examples using an autoencoder-based model. It first extracts transferable intra-class deformations (deltas) between same-class pairs of training examples, then applies them to a few examples of a new class to synthesize samples from that class. Authors show that Delta-Encoder can learn transferable deformations from different source classes which can be used to generate examples for unseen classes. While the authors used Delta-Encoder to generate examples for unseen classes, in our experiments, for FSI, we also use the examples from the target class to the train both the feature extractor and the Delta-Encoder along with all other examples. Then we generate new examples for the target category using trained delta encoder. For data generation, we try two different approaches to select a source sentence pair.

1. DeltaR: Sample a pair of sentences $\left(X_{i}, X_{j}\right)$ from a randomly selected class. DELTAR applies deltas from multiple source categories to synthesize new examples.

2. DeltaS: Sample a pair of sentences $\left(X_{i}, X_{j}\right)$ from the target category. DELTAS applies deltas from the same target category.

The encoder and decoder sub-networks are implemented as multi-layer perceptrons with a single hidden layer of 512 units, where each layer is followed by a leaky ReLU activation $(\max (x, 0.2 *$ $x)$ ). The encoder output $Z$ is 16 -dimensional. L1 loss is used as reconstruction loss. Adam optimizer is used with a learning rate of $10-3$. A high dropout with a $50 \%$ rate is applied to all layers, to avoid the model memorizing examples.

\section{Experiment}

\subsection{Datasets}

We evaluate different FDA techniques on two public benchmark datasets, SNIPS (Coucke et al., 2018), and Facebook Dialog corpus (FBDialog) (Gupta et al., 2018). For SNIPS dataset, we use train, dev and test split provided by (Goo et al., 2018) ${ }^{2}$.

SNIPS dataset contains 7 intents which are collected from the Snips personal voice assistant. The training, development and test sets contain 13, 084, 700 and 700 utterances, respectively. FBDialog has utterances that are focused on navigation, events, and navigation to events. FBDialog dataset also contains utterances with multiple intents as the root node. For our experiment, we exclude such utterances by removing utterances with COMBINED intent root node. This leads to 31, 218 training, 4, 455 development and 9, 019 testset utterances. Note that while SNIPS is a balanced dataset, FBDialog dataset is highly imbalanced with a maximum 8,860 and a minimum of 4 training examples per intent.

\subsection{Simulating Few-Shot Integration}

In virtual assistants, often a new intent development starts with very limited training data. To simulate the integration of a new intent, we randomly sample $k$ seed training examples from the new intent, referred to as target intent, and keep all the data from other intents. We also remove the target intent data from the development set. We train the feature extractor on the resulting training data, and then generate 100, 512 examples using different augmentation methods for the target intent. To account for random fluctuations in the results, we repeat this process 10 times for a given target intent and report the average accuracy with the standard deviation. In all experiments, models are evaluated on the full test set.

\footnotetext{
${ }^{2}$ https://github.com/MiuLab/SlotGated-SLU
} 


\begin{tabular}{|c|c|c|c|}
\hline Size & Method & SNIPS & FBDialog \\
\hline \multicolumn{2}{|c|}{ No Augmentation } & $98.14(0.42)$ & $94.99(0.18)$ \\
\hline \multirow{7}{*}{$5 \%$} & UPSAMPLE & $98.14(0.47)$ & $95.01(0.16)$ \\
\hline & PERTURB & $\mathbf{9 8 . 2 6}(0.40)$ & $94.98(0.19)$ \\
\hline & LINEAR & $98.14(0.45)$ & $95.02(0.21)$ \\
\hline & EXTRA & $98.14(0.45)$ & $95.02(0.20)$ \\
\hline & CVAE & $98.14(0.45)$ & $94.98(0.24)$ \\
\hline & DELTAR & $98.23(0.46)$ & $95.00(0.22)$ \\
\hline & DELTAS & $\mathbf{9 8 . 2 6}(0.42)$ & $95.00(0.20)$ \\
\hline \multirow{7}{*}{$10 \%$} & UPSAMPLE & $98.14(0.47)$ & $94.94(0.18)$ \\
\hline & PERTURB & $98.23(0.41)$ & $94.98(0.24)$ \\
\hline & LINEAR & $98.09(0.50)$ & $95.02(0.18)$ \\
\hline & EXTRA & $98.11(0.49)$ & $95.01(0.19)$ \\
\hline & CVAE & $98.20(0.42)$ & $94.99(0.26)$ \\
\hline & DELTAR & $\mathbf{9 8 . 2 6}(0.42)$ & $94.99(0.21)$ \\
\hline & DELTAS & $98.23(0.42)$ & $94.97(0.22)$ \\
\hline \multirow{7}{*}{$20 \%$} & UPSAMPLE & $98.14(0.45)$ & $95.02(0.12)$ \\
\hline & PERTURB & $98.14(0.44)$ & $94.99(0.20)$ \\
\hline & LINEAR & $98.17(0.43)$ & $95.05(0.23)$ \\
\hline & EXTRA & $98.14(0.45)$ & $95.07(0.11)$ \\
\hline & CVAE & $98.11(0.44)$ & $94.98(0.23)$ \\
\hline & DELTAR & $\mathbf{9 8 . 2 6}(0.40)$ & $95.08(0.19)$ \\
\hline & DELTAS & $98.20(0.46)$ & $95.04(0.22)$ \\
\hline
\end{tabular}

Table 1: IC accuracy on SNIPS and Facebook dataset with all training data, reported as mean (SD).

\section{Results and Discussion}

\subsection{FDA For Data-Rich Classification}

For both datasets, we generate $5 \%, 10 \%$, and $20 \%$ examples using different FDA methods. Then, we train a classifier using both generated as well as real data. Table 1 shows that augmenting data in feature space provides only minor improvements in classification accuracy. In particular, on SNIPS dataset, PERTUb and DELTAR improve accuracy from 98.14 to 98.26. On FBDialog dataset, DeltaR provides a minor gain, 95.02 to 95.08 over upsample baseline.

\subsection{Impact Of The Number Of Seed Examples}

To understand the impact of the number of seed examples, we vary it to $5,10,15,20,25$, and 30 for SNIPS's AddToPlaylist. For each experiment, we generate 100 examples using different FDA methods. Figure 2 shows that as the number of seed examples increases, the accuracy of the model goes up. We also observe that for a few seed examples 5 - 15, LINEAR outperforms other FSA methods. Finally, gains are less significant after 30 seed examples.

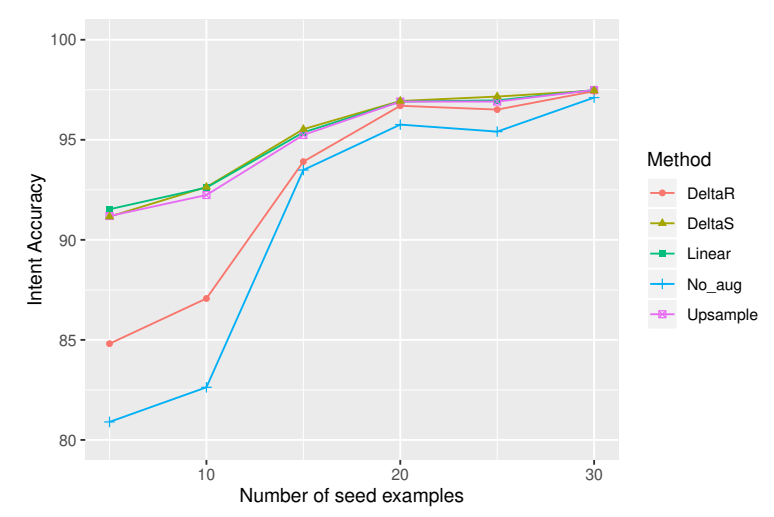

Figure 2: IC accuracy on SNIPS's AddToPlaylist intent with varying number of seed examples. 100 examples are generated using different FDA techniques. As indicated by the accuracy trend, increasing the seed examples leads to better performance.

\subsection{Few-Shot Integration}

We simulate FSI IC for all 7 intents of SNIPS dataset. For FBDialog dataset, we run simulations on the six largest intents, viz. GetDirections, GetDistance, GetEstimatedArrival, GetEstimatedDuration, GetInfoTraffic, and GetEvent. Since, BERT generalizes well with just 30 examples, to compare the effectiveness of different FDA methods, we use 10 seed examples in FSI simulations. For each intent, we select $k=10$ seed training examples and use all training data for other intents.

Table 2 shows average accuracy for all intents' FSI simulations. Results on individual intent's FSI simulations can be found in Appendix's Table 5 and Table 6. On both datasets, all FDA methods improve classification accuracy over no augmentation baseline. Also, UPSAMPLE provides huge gains over no augmentation baseline. Additionally, on both datasets, with 512 augmented examples, LINEAR and DELTAS works better than PERTURB and UPSAMPLE.

\subsection{Upsampling: Text Space vs Latent Space}

In this section, we explore how upsampling in text space impacts performances as it is supposed to both improve the feature extractor and the linear classifier, compared to UPSAMPLE. To investigate whether upsampling in text space helps FDA, we upsampled the 10 seed examples to 100 and repeat the FSI experiments on all 7 intents of the SNIPS dataset. Table 3 shows the mean accuracy of all 7 intents FSI simulations results for different FDA techniques. FSI simulations scores for individual intents can be found in Appendix's Table 7. We 


\begin{tabular}{llll}
\hline$\#$ & Method & SNIPS & FBDialog \\
\hline No Augmentation & $87.46(2.87)$ & $81.29(0.11)$ \\
\hline \multirow{6}{*}{100} & UPSAMPLE & $94.26(1.66)$ & $\mathbf{8 4 . 3 4}(1.84)$ \\
& PERTURB & $94.18(1.74)$ & $84.04(1.95)$ \\
& CVAE & $94.10(1.83)$ & $84.10(1.94)$ \\
& LINEAR & $\mathbf{9 4 . 3 6}(1.69)$ & $84.31(1.9)$ \\
& EXTRA & $94.30(1.68)$ & $84.13(1.83)$ \\
& DELTAR & $91.32(3.12)$ & $81.97(0.76)$ \\
& DELTAS & $94.28(1.92)$ & $83.50(1.92)$ \\
\hline \multirow{6}{*}{ 512 } & UPSAMPLE & $95.68(0.86)$ & $89.03(0.99)$ \\
& PERTURB & $95.65(0.92)$ & $89.02(0.99)$ \\
CVAE & $95.46(1.03)$ & $88.71(1.09)$ \\
& LINEAR & $\mathbf{9 5 . 8 7}(0.87)$ & $\mathbf{8 9 . 3 0}(1.03)$ \\
& EXTRA & $95.82(0.89)$ & $89.21(0.99)$ \\
& DELTAR & $95.33(1.56)$ & $87.28(1.46)$ \\
& DELTAS & $\mathbf{9 5 . 8 8}(1.04)$ & $89.15(1.12)$ \\
\hline
\end{tabular}

Table 2: Average IC accuracy for all intents' FSI simulations on SNIPS and FBDialog dataset. For each simulation, $k=10$ seed examples are used for target intent. Scores are reported as mean (SD). Refer to Appendix's Table 5 and Table 6 for individual intents' results.

observe that upsampling in text space improves the no augmentation baseline for all intents. The mean accuracy score improves from 87.46 to 94.38 . We also observe that different FDA techniques further improve model accuracy. Interestingly, upsampling in text space helps DeltaR the most. Surprisingly, upsampling in latent space provides better performance than upsampling in the text space. In particular, without upsampling the seed examples to learn the feature extractor, the best score is 95.88 for DELTAS, whereas with text space upsampling the best score decreases to 94.88. This decrease in performance is only seen with BERT and not with the Bi-LSTM feature extractor (see Table 4). We hypothesize that upsampling text data leads to BERT overfitting the target category which results in less generalized sentence representations. Overall, we found that augmentation in the latent space seems to work better with BERT, and is more effective than text space upsampling.

\subsection{Effect Of The Pre-trained BERT Encoder}

In FSI setting, Fine-Tuned BERT model provides very good generalization performance. For example, for SNIPS's RateBookIntent (column Book in Table 5), it yields $96.81 \%$ accuracy. Overall for BERT representations, LINEAR and DELTAS augmentation methods provide the best accuracy.

\begin{tabular}{|c|c|c|}
\hline$\#$ & Method & Overall Mean \\
\hline No & ugmentation & $94.38(1.23)$ \\
\hline \multirow{7}{*}{100} & UPSAMPLE & $94.53(1.12)$ \\
\hline & PERTURb & $94.52(1.18)$ \\
\hline & CVAE & $94.53(1.18)$ \\
\hline & LINEAR & $94.53(1.12)$ \\
\hline & EXTRA & $94.53(1.13)$ \\
\hline & DELTAR & $94.62(1.16)$ \\
\hline & DELTAS & $94.57(1.14)$ \\
\hline \multirow{7}{*}{512} & UPSAMPLE & $94.67(1.11)$ \\
\hline & PERTURB & $94.68(1.14)$ \\
\hline & CVAE & $94.73(1.11)$ \\
\hline & LINEAR & $94.67(1.11)$ \\
\hline & EXTRA & $94.67(1.11)$ \\
\hline & DELTAR & $\mathbf{9 4 . 8 8}(1.12)$ \\
\hline & DELTAS & $94.74(1.12)$ \\
\hline
\end{tabular}

Table 3: IC accuracy on SNIPS dataset in the FSI setting, reported as mean $(S D)$. The 10 seed examples are upsampled to 100 to train the feature extractor. Refer to Appendix's Table 7 for individual intents' results.

To investigate whether these augmentation improvements can be generalized to other sentence encoders, we experiment with a Bi-LSTM sentence encoder. For feature learning, we use a 1layer Bi-LSTM encoder followed by a single layer softmax classifier. In our experiments, we use 128 as hidden units and 300 dimension Glove embeddings. For SNIPS dataset, we use 10 examples of AddToPlaylist intent and for FB Dialog dataset, we use 10 examples of GetDirections intent.

Table 4 shows intent accuracy for SNIPS and Facebook datasets. We find that, unlike BERT, in the FSI setting, the Bi-LSTM encoder provides a lower accuracy. In contrast to BERT FSI experiments, DELTAS performs worse than the UPSAMPLE and PERTURB baselines. The main reason is that Delta-Encoder's performance relies on a good feature extractor and with 10 seed examples, the Bi-LSTM encoder fails to learn good sentence representations. To improve representation learning, we upsample 10 utterances to 100 and then train the feature extractor. Upsampling in text space improves the performance of both delta encoder methods, DeltaS, and DelTAR. Moreover, for both SNIPS's AddToPlayList and FBDialog's GetDirections intent, DELTAR outperforms all other FDA methods. 


\begin{tabular}{clllll}
\hline Size & Method & \multicolumn{2}{l}{ SNIPS's AddToPlaylist } & \multicolumn{2}{l}{ FBDialog's GetDirections } \\
\hline seed examples $(k)$ & 10 & $100^{*}$ & 10 & $100^{*}$ \\
\hline No Augmentation & $80.07(2.08)$ & $90.17(1.39)$ & $87.44(0.12)$ & $87.94(0.32)$ \\
\hline UPSAMPLE & $\mathbf{8 8 . 2 7}(1.74)$ & $90.61(1.52)$ & $88.01(0.26)$ & $88.17(0.32)$ \\
PERTURB & $88.03(1.52)$ & $90.86(1.39)$ & $88.01(0.32)$ & $88.25(0.31)$ \\
LINEAR & $88.14(1.62)$ & $91.06(1.58)$ & $88.05(0.25)$ & $88.26(0.32)$ \\
100 & EXTRA & $88.09(1.57)$ & $90.74(1.57)$ & $\mathbf{8 8 . 1 0}(0.29)$ & $88.20(0.3)$ \\
CVAE & $\mathbf{8 8 . 2 7}(2.08)$ & $90.90(1.69)$ & $88.04(0.24)$ & $88.17(0.32)$ \\
DELTAR & $82.23(2.21)$ & $\mathbf{9 1 . 4 6}(1.19)$ & $87.60(0.23)$ & $\mathbf{8 8 . 7 5}(0.43)$ \\
DELTAS & $84.4(2.74)$ & $91.07(1.44)$ & $88.02(0.22)$ & $88.57(0.36)$ \\
\hline UPSAMPLE & $91.41(1.03)$ & $91.61(1.4)$ & $88.68(0.49)$ & $88.40(0.35)$ \\
PERTURB & $\mathbf{9 1 . 4 6}(0.99)$ & $91.73(1.32)$ & $88.89(0.57)$ & $88.56(0.39)$ \\
LINEAR & $91.20(1.28)$ & $91.41(1.52)$ & $88.97(0.65)$ & $88.47(0.33)$ \\
512 & EXTRA & $91.26(1.22)$ & $91.57(1.55)$ & $88.85(0.61)$ & $88.48(0.37)$ \\
CVAE & $91.39(0.94)$ & $91.44(1.2)$ & $89.02(0.52)$ & $88.48(0.4)$ \\
DELTAR & $87.09(2.75)$ & $\mathbf{9 2 . 9 7}(1.2))$ & $88.61(0.35)$ & $\mathbf{8 9 . 7 0}(0.53)$ \\
DELTAS & $89.34(1.48)$ & $92.00(1.25)$ & $\mathbf{8 9 . 3 4}(0.4)$ & $89.09(0.51)$ \\
\hline
\end{tabular}

Table 4: IC accuracy on SNIPS's AddToPlaylist and FBDialog's GetDirections in the FSI setting, reported as mean (SD). A 1-layer Bi-LSTM model is used as a feature extractor. $100^{*}$ represents 10 seed examples are upsampled to 100 to train the feature extractor.

\subsection{Is Delta-Encoder Effective On Text?}

While on few-shot image classification, DeltaEncoder provides excellent generalization performance (Schwartz et al., 2018) on unseen classes, on text classification, its performance is heavily dependent on the feature extractor. We observe that in most cases, DELTAR performs worse than DELTAS which suggests that unlike for few-shot image classification, Delta-Encoder fails to learn variations which can be applied to a different category. In addition, in FSI with BERT encoder, DELTAS performance is close to LINEAR. This indicates that in the low-data regime, simple subtraction between BERT sentence representations is a good proxy to learn intra-class variations. Upsampling data in text space improves Delta-Encoder performance for both BERT and Bi-LSTM encoders. As shown in Table 3, with upsampling in text space, DELTAR performs better than any other FDA method.

\subsection{Qualitative Evaluation}

We observe significant accuracy improvements in all FSI experiments for all FDA methods. Since UPSAMPLE and PERTURB also provide significant gains, it seems that most of the gains come from the fact that we are adding more data. However, in the FSI setting, LINEAR and DELTAS method consistently perform better than both UPSAMPLE and Perturb, which indicates that these methods generate more relevant data than just noise, and redundancy. Here, we focus on visualizing generated examples from LINEAR, DELTAS and DELTAR methods using t-SNE.
Figure 3 shows visualizations for SNIPS's AddToPlaylist generated sentence representations using different FDA methods. We use 10 seed examples of AddToPlaylist and use BERT as sentence encoder. While data generated by LINEAR and EXTRA are close to the real examples, DeltaS and DELTAR generated examples form two different clusters. Since, Delta-Encoder performance improves when seed examples are upsampled in text space, we plot sentence examples from upsampled data.

Figure 4 shows that when 10 seed examples are upsampled to 100, DeltaS cluster moves closer to the seed examples, and while most of the DELTAR generated data forms a separate cluster, a few of the generated examples are close to the seed examples. Since, in experiments with upsampled text examples, DELTAR performs better than other FDA methods, we hypothesize that DELTAR increases the amount of variability within the dataset by generating diverse examples which leads to a more robust model.

\section{Conclusion and Future Work}

In this paper, we investigate six FDA methods including Upsample, Perturb, CVAE, DeltaEncoder, EXTRA, and LINEAR to augment training data. We show that FDA works better when combined with transfer learning and provides an effective way of bootstrapping an intent classifier for new classes. As expected, all FDA methods become less effective when the number of seed examples increases and provides minor gains in the full-data regime. Through comparing methods on two public datasets, our results show that LINEAR is a competitive baseline for FDA in FSI setting, especially when combined with transfer learning (BERT).

Additionally, we provide empirical evidence that in few-shot integration setting, feature space augmentation combined with BERT provides better performance than widely used text space upsampling. Given that pre-trained language models provide state of the art performance on several NLP tasks, we find this result to be in particular encouraging, as it shows potential for applying FDA methods to other NLP tasks.

Our experiments on Delta-Encoder also shows that unlike few-shot image classification, DeltaEncoder fails to learn transferable intra-class variations. This result emphasizes that methods pro- 


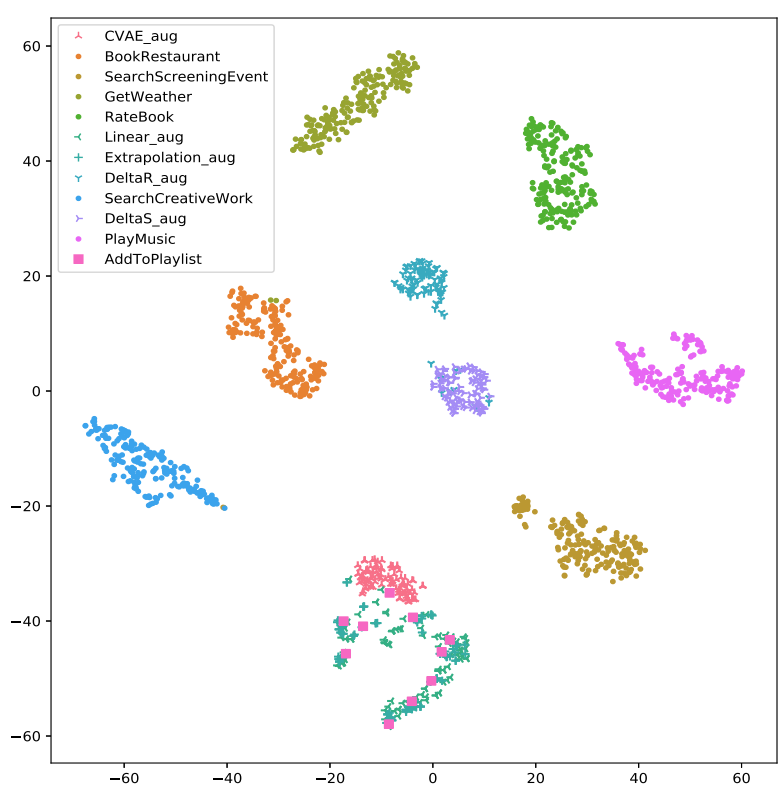

Figure 3: 10 seed examples

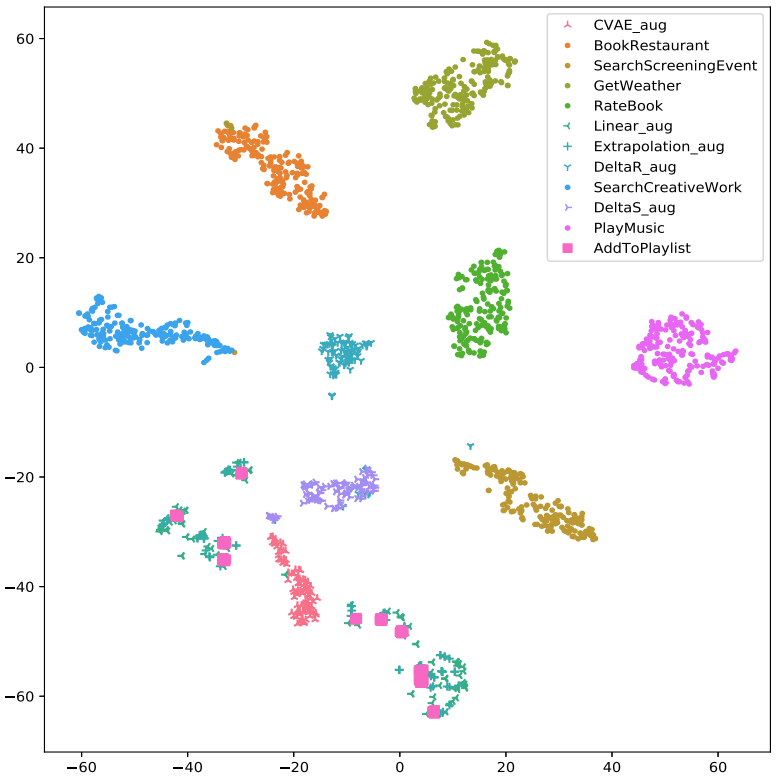

Figure 4: 10 seed examples are upsampled to 100

Figure 5: t-SNE visualization of different data augmentation methods for AddToPlaylist intent. BERT encoder is used to learn sentence representations.

viding improvements in computer vision domain might not produce similar gains on NLP tasks, thus underlining the need to develop data augmentation methods specific to NLP tasks.

\section{References}

Anthreas Antoniou, Amos Storkey, and Harrison Edwards. 2018. Data augmentation generative adversarial networks.

Nitesh V Chawla, Kevin W Bowyer, Lawrence O Hall, and W Philip Kegelmeyer. 2002. Smote: synthetic minority over-sampling technique. Journal of artificial intelligence research.

Yu Cheng, Mo Yu, Xiaoxiao Guo, and Bowen Zhou. 2019. Few-shot learning with meta metric learners.

Eunah Cho, He Xie, and William M Campbell. 2019a. Paraphrase generation for semi-supervised learning in nlu. In Proceedings of the Workshop on Methods for Optimizing and Evaluating Neural Language Generation.

Eunah Cho, He Xie, John Lalor, Varun Kumar, and William M Campbell. 2019b. Efficient semisupervised learning for natural language understanding by optimizing diversity. In IEEE Automatic Speech Recognition and Understanding Workshop.

Alice Coucke, Alaa Saade, Adrien Ball, Théodore Bluche, Alexandre Caulier, David Leroy, Clément Doumouro, Thibault Gisselbrecht, Francesco Caltagirone, Thibaut Lavril, et al. 2018. Snips voice platform: an embedded spoken language understanding system for private-by-design voice interfaces. arXiv preprint arXiv:1805.10190.

Jacob Devlin, Ming-Wei Chang, Kenton Lee, and Kristina Toutanova. 2018. Bert: Pre-training of deep bidirectional transformers for language understanding. arXiv preprint arXiv:1810.04805.

Terrance Devries and Graham W. Taylor. 2017. Dataset augmentation in feature space. ArXiv, abs/1702.05538.

Andrew Estabrooks, Taeho Jo, and Nathalie Japkowicz. 2004. A multiple resampling method for learning from imbalanced data sets. Computational intelligence.

Chelsea Finn, Pieter Abbeel, and Sergey Levine. 2017. Model-agnostic meta-learning for fast adaptation of deep networks. In Proceedings of the 34th International Conference on Machine Learning. JMLR.org.

Chih-Wen Goo, Guang Gao, Yun-Kai Hsu, Chih-Li Huo, Tsung-Chieh Chen, Keng-Wei Hsu, and YunNung Chen. 2018. Slot-gated modeling for joint slot filling and intent prediction. In Proceedings of the North American Chapter of the Association for Computational Linguistics.

Hongyu Guo, Yongyi Mao, and Richong Zhang. 2019. Augmenting data with mixup for sentence classification: An empirical study. arXiv preprint arXiv:1905.08941.

Rahul Gupta. 2019. Data augmentation for low resource sentiment analysis using generative adversarial networks. In IEEE International Conference on Acoustics, Speech and Signal Processing. 
Sonal Gupta, Rushin Shah, Mrinal Mohit, Anuj Kumar, and Mike Lewis. 2018. Semantic parsing for task oriented dialog using hierarchical representations. arXiv preprint arXiv:1810.07942.

Kelvin Guu, Tatsunori B Hashimoto, Yonatan Oren, and Percy Liang. 2018. Generating sentences by editing prototypes. Transactions of the Association of Computational Linguistics.

Bharath Hariharan and Ross Girshick. 2017. Lowshot visual recognition by shrinking and hallucinating features. In Proceedings of the International Conference on Computer Vision.

Yutai Hou, Yijia Liu, Wanxiang Che, and Ting Liu. 2018. Sequence-to-sequence data augmentation for dialogue language understanding. arXiv preprint arXiv:1807.01554.

Xiang Jiang, Mohammad Havaei, Gabriel Chartrand, Hassan Chouaib, Thomas Vincent, Andrew Jesson, Nicolas Chapados, and Stan Matwin. 2018. Attentive task-agnostic meta-learning for few-shot text classification.

Diederik P Kingma and Jimmy Ba. 2014. Adam: A method for stochastic optimization. arXiv preprint arXiv:1412.6980.

Diederik P Kingma and Max Welling. 2013. Autoencoding variational bayes. arXiv preprint arXiv:1312.6114.

Gregory Koch, Richard Zemel, and Ruslan Salakhutdinov. 2015. Siamese neural networks for one-shot image recognition. In ICML Deep Learning Workshop.

Gakuto Kurata, Bing Xiang, and Bowen Zhou. 2016. Labeled data generation with encoder-decoder lstm for semantic slot filling. In INTERSPEECH.

Zhenguo Li, Fengwei Zhou, Fei Chen, and Hang Li. 2017. Meta-sgd: Learning to learn quickly for fewshot learning. arXiv preprint arXiv:1707.09835.

Swee Kiat Lim, Yi Loo, Ngoc-Trung Tran, Ngai-Man Cheung, Gemma Roig, and Yuval Elovici. 2018. Doping: Generative data augmentation for unsupervised anomaly detection with gan. In 2018 IEEE International Conference on Data Mining.

Akshay Mehrotra and Ambedkar Dukkipati. 2017. Generative adversarial residual pairwise networks for one shot learning.

Stanislav Peshterliev, John Kearney, Abhyuday Jagannatha, Imre Kiss, and Spyros Matsoukas. 2018. Active learning for new domains in natural language understanding. arXiv preprint arXiv:1810.03450.

Matthew E Peters, Mark Neumann, Mohit Iyyer, Matt Gardner, Christopher Clark, Kenton Lee, and Luke Zettlemoyer. 2018. Deep contextualized word representations. arXiv preprint arXiv:1802.05365.
Alec Radford, Karthik Narasimhan, Tim Salimans, and Ilya Sutskever. 2018. Improving language understanding by generative pre-training.

Sachin Ravi and Hugo Larochelle. 2016. Optimization as a model for few-shot learning.

Oren Rippel, Manohar Paluri, Piotr Dollar, and Lubomir Bourdev. 2015. Metric learning with adaptive density discrimination. arXiv preprint arXiv:1511.05939.

Eli Schwartz, Leonid Karlinsky, Joseph Shtok, Sivan Harary, Mattias Marder, Abhishek Kumar, Rogerio Feris, Raja Giryes, and Alex Bronstein. 2018. Deltaencoder: an effective sample synthesis method for few-shot object recognition. In Advances in Neural Information Processing Systems.

Jake Snell, Kevin Swersky, and Richard Zemel. 2017. Prototypical networks for few-shot learning. In I. Guyon, U. V. Luxburg, S. Bengio, H. Wallach, R. Fergus, S. Vishwanathan, and R. Garnett, editors, Advances in Neural Information Processing Systems.

Oriol Vinyals, Charles Blundell, Tim Lillicrap, Koray Kavukcuoglu, and Daan Wierstra. 2016. Matching networks for one shot learning. In D. D. Lee, M. Sugiyama, U. V. Luxburg, I. Guyon, and R. Garnett, editors, Advances in Neural Information Processing Systems.

Yu-Xiong Wang, Ross Girshick, Martial Hebert, and Bharath Hariharan. 2018. Low-shot learning from imaginary data. In Proceedings of the IEEE Conference on Computer Vision and Pattern Recognition.

Kang Min Yoo, Youhyun Shin, and Sang goo Lee. 2018. Data augmentation for spoken language understanding via joint variational generation.

Mo Yu, Xiaoxiao Guo, Jinfeng Yi, Shiyu Chang, Saloni Potdar, Yu Cheng, Gerald Tesauro, Haoyu Wang, and Bowen Zhou. 2018. Diverse few-shot text classification with multiple metrics. In Proceedings of the North American Chapter of the Association for Computational Linguistics.

Ruixiang Zhang, Tong Che, Zoubin Ghahramani, Yoshua Bengio, and Yangqiu Song. 2018. Metagan: An adversarial approach to few-shot learning. In Advances in Neural Information Processing Systems.

Zijian Zhao, Su Zhu, and Kai Yu. 2019. Data augmentation with atomic templates for spoken language understanding. arXiv preprint arXiv:1908.10770.

\section{A FSI experiment results for all intents}

In all tables, individual columns represent FSI results for an intent, and Overall Mean column, provides average accuracy for all intents' FSI simulations. 


\begin{tabular}{|c|c|c|c|c|c|c|c|c|c|}
\hline$\#$ & Method & Playlist & Restaurant & Weather & Music & Book & Work & Event & Overall Mean \\
\hline No & Augmentation & $82.63(5.11)$ & $87.86(3.53)$ & $84.51(1.3)$ & $88.07(2.37)$ & $96.81(2.94)$ & $85.14(1.53)$ & $87.19(3.31)$ & $87.46(2.87)$ \\
\hline \multirow{7}{*}{100} & UPSAMPLE & $92.24(2.96)$ & $97.7(0.67)$ & $96.44(0.75)$ & $94.57(1.1)$ & $97.96(0.82)$ & $89.61(3.01)$ & $91.26(2.35)$ & $94.26(1.66)$ \\
\hline & PERTURB & 93.09(2.55) & $97.41(0.92)$ & $96.07(1.35)$ & $94.39(1.13)$ & $97.86(0.93)$ & $89.36(2.76)$ & $91.09(2.53)$ & $94.18(1.74)$ \\
\hline & CVAE & $92.4(3.66)$ & $97.47(0.67)$ & $96.49(1.07)$ & $94.36(1.26)$ & $97.71(1.1)$ & $89.1(2.79)$ & $91.2(2.22)$ & $94.1(1.83)$ \\
\hline & LINEAR & $92.61(3.02)$ & $97.74(0.67)$ & $96.44(0.77)$ & $94.63(1.18)$ & $\mathbf{9 7 . 9 7}(0.78)$ & 89.61(3.05) & $91.53(2.34)$ & 94.36(1.69) \\
\hline & EXTRA & $92.36(3.0)$ & $97.74(0.66)$ & $96.41(0.77)$ & $94.6(1.18)$ & $97.97(0.78)$ & $89.47(3.11)$ & $91.51(2.3)$ & $94.3(1.68)$ \\
\hline & DELTAR & $87.07(4.67)$ & $93.57(4.07)$ & $91.0(4.23)$ & $94.87(1.28)$ & $97.66(1.42)$ & $85.97(2.34)$ & $89.11(3.84)$ & $91.32(3.12)$ \\
\hline & DELTAS & $92.64(4.49)$ & $\mathbf{9 7 . 7 6}(0.7)$ & $96.41(1.25)$ & $\mathbf{9 4 . 9 9}(0.92)$ & $97.83(0.99)$ & $9(2.69)$ & $\mathbf{9 1 . 6 4}(2.36)$ & $94.28(1.92)$ \\
\hline \multirow{7}{*}{512} & UPSAMPLE & $95.3(1.09)$ & $98.0(0.64)$ & $97.63(0.34)$ & $95.57(0.87)$ & $98.03(0.55)$ & $92.0(1.49)$ & $93.26(1.05)$ & $95.68(0.86)$ \\
\hline & PERTURB & $95.33(1.2)$ & $97.94(0.6)$ & $97.6(0.44)$ & $95.5(0.91)$ & $97.91(0.55)$ & $92.03(1.78)$ & $93.21(0.99)$ & $95.65(0.92)$ \\
\hline & CVAE & $95.46(1.12)$ & $97.89(0.62)$ & $97.54(0.43)$ & $95.36(1.02)$ & $97.93(0.7)$ & $91.34(2.17)$ & $92.73(1.19)$ & $95.46(1.03)$ \\
\hline & LINEAR & $95.39(1.1)$ & $98.0(0.64)$ & $97.67(0.36)$ & $95.74(0.89)$ & $98.04(0.5)$ & $\mathbf{9 2 . 6 1 ( 1 . 4 7 )}$ & $93.66(1.13)$ & 95.87(0.87) \\
\hline & EXTRA & $95.36(1.17)$ & $98.0(0.64)$ & $97.66(0.37)$ & $95.74(0.88)$ & $98.04(0.5)$ & $92.29(1.52)$ & $93.63(1.17)$ & $95.82(0.89)$ \\
\hline & DELTAR & $95.36(1.74)$ & $97.81(0.69)$ & $97.6(0.44)$ & $95.9(0.97)$ & $97.74(1.02)$ & $90.27(3.44)$ & $92.61(2.64)$ & $95.33(1.56)$ \\
\hline & DELTAS & 95.66(1.18) & $97.96(0.59)$ & $\mathbf{9 7 . 8}(0.45)$ & $\mathbf{9 5 . 9 1}(0.88)$ & $97.91(0.74)$ & $92.26(2.57)$ & 93.66(0.86) & 95.88(1.04) \\
\hline
\end{tabular}

Table 5: IC accuracy on SNIPS dataset in the FSI setting $(k=10)$, reported as mean (SD).

\begin{tabular}{|c|c|c|c|c|c|c|c|c|}
\hline \# & Method & Directions & Distance & Arrival & Duration & Traffic & Event & Overall Mean \\
\hline No $A$ & ugmentation & $89.61(0.1)$ & $89.94(0.09)$ & $90.56(0.12)$ & $81.74(0.13)$ & $68.5(0.13)$ & $67.39(0.11)$ & $81.29(0.11)$ \\
\hline \multirow{7}{*}{100} & UPSAMPLE & $89.89(0.27)$ & 93.64(0.87) & $92.95(0.57)$ & $84.28(3.45)$ & $68.99(0.49)$ & 76.26(5.41) & 84.34(1.84) \\
\hline & PERTURB & $89.82(0.24)$ & $93.58(0.84)$ & $92.81(0.55)$ & 84.81(3.77) & 69.15(0.68) & $74.07(5.6)$ & $84.04(1.95)$ \\
\hline & CVAE & $89.91(0.32)$ & $93.46(0.77)$ & $92.7(0.67)$ & $84.45(3.52)$ & $69.11(0.9)$ & 74.94(5.49) & 84.1(1.94) \\
\hline & LINEAR & $\mathbf{8 9 . 9 3}(0.24)$ & $\mathbf{9 3 . 6 5}(0.88)$ & $\mathbf{9 2 . 9 8}(0.57)$ & $84.2(3.44)$ & $68.96(0.51)$ & 76.12 & 84.3 \\
\hline & EXTRA & $89.88(0.27)$ & $93.61(0.89)$ & $92.96(0.59)$ & $84.21(3.43)$ & 68.94(0.46) & 75.18 & $84.13(1.83)$ \\
\hline & DELTAR & $89.64(0.11)$ & $92.57(1.3)$ & $90.79(0.37)$ & $81.72(0.12)$ & $68.48(0.08)$ & $68.63(2.59)$ & $81.97(0.76)$ \\
\hline & DELTAS & $89.88(0.34)$ & $93.68(0.72)$ & $92.6(0.76)$ & $83.88(3.2)$ & 68.93(0.67) & $72.05(5.83)$ & $83.5(1.92)$ \\
\hline \multirow{7}{*}{512} & UPSAMPLE & $91.93(0.48)$ & $94.58(0.34)$ & $93.99(0.31)$ & $92.56(0.72)$ & $75.84(2.19)$ & $85.27(1.87)$ & $89.03(0.99)$ \\
\hline & PERTURB & $91.78(0.49)$ & $94.58(0.43)$ & $94.02(0.25)$ & $92.53(0.87)$ & 76.0(2.27) & $85.22(1.61)$ & $89.02(0.99)$ \\
\hline & CVAE & $91.85(0.52)$ & $94.57(0.39)$ & $94.0(0.34)$ & $92.45(0.92)$ & 74.91(2.73) & $84.5(1.61)$ & $88.71(1.09)$ \\
\hline & LINEAR & $92.14(0.66)$ & $94.6(0.35)$ & $94.05(0.32)$ & $\mathbf{9 2 . 7 8}(0.67)$ & 76.0(2.49) & $86.22(1.7)$ & 89.3(1.03) \\
\hline & EXTRA & $\mathbf{9 2 . 1 1}(0.57)$ & $94.61(0.35)$ & $94.04(0.29)$ & $92.72(0.7)$ & $75.79(2.45)$ & $85.98(1.58)$ & $89.21(0.99)$ \\
\hline & DELTAR & $90.43(0.55)$ & $94.54(0.35)$ & $93.8(0.3)$ & $86.64(4.38)$ & 71.68(1.46) & $\mathbf{8 6 . 5 5}(1.75)$ & $87.28(1.46)$ \\
\hline & DELTAS & $91.83(0.47)$ & $94.66(0.4)$ & 94.08(0.24) & $92.31(1.45)$ & $75.81(2.1)$ & $86.23(2.08)$ & $89.15(1.12)$ \\
\hline
\end{tabular}

Table 6: IC accuracy on FBDialog dataset in the FSI setting $(k=10)$, reported as mean $(S D)$.

\begin{tabular}{llllllllll}
\hline$\#$ & Method & Playlist & Restaurant & Weather & Music & Book & Work & Event & Overall Mean \\
\hline No Augmentation & $96.0(1.69)$ & $95.39(1.59)$ & $96.41(1.18)$ & $93.1(1.38)$ & $97.79(0.77)$ & $88.46(1.14)$ & $93.49(0.87)$ & $94.38(1.23)$ \\
\hline UPSAMPLE & $96.0(1.57)$ & $95.87(1.26)$ & $\mathbf{9 6 . 5 1}(1.04)$ & $93.19(1.25)$ & $97.83(0.7)$ & $88.63(1.21)$ & $93.7(0.83)$ & $94.53(1.12)$ \\
& PERTURB & $96.1(1.64)$ & $95.7(1.23)$ & $96.43(1.28)$ & $93.33(1.1)$ & $97.8(0.77)$ & $88.56(1.32)$ & $93.7(0.9)$ & $94.52(1.18)$ \\
100 & CVAE & $96.07(1.46)$ & $\mathbf{9 5 . 9 1 ( 1 . 4 3 )}$ & $96.43(1.31)$ & $93.2(1.15)$ & $97.83(0.78)$ & $88.63(1.28)$ & $93.66(0.86)$ & $94.53(1.18)$ \\
& LINEAR & $96.0(1.57)$ & $95.89(1.26)$ & $\mathbf{9 6 . 5 1}(1.04)$ & $93.19(1.25)$ & $97.83(0.7)$ & $88.63(1.21)$ & $93.7(0.83)$ & $94.53(1.12)$ \\
& EXTRA & $96.0(1.57)$ & $95.84(1.3)$ & $\mathbf{9 6 . 5 1}(1.04)$ & $93.19(1.25)$ & $97.83(0.7)$ & $88.63(1.21)$ & $93.7(0.83)$ & $94.53(1.13)$ \\
& DELTAR & $96.09(1.51)$ & $95.74(1.46)$ & $96.44(1.29)$ & $\mathbf{9 3 . 5 6}(0.95)$ & $\mathbf{9 7 . 8 6}(0.75)$ & $\mathbf{8 8 . 7 9}(1.25)$ & $\mathbf{9 3 . 8 6}(0.93)$ & $\mathbf{9 4 . 6 2 ( 1 . 1 6 )}$ \\
DELTAS & $\mathbf{9 6 . 1 1}(1.52)$ & $95.69(1.44)$ & $96.46(1.29)$ & $93.44(0.93)$ & $\mathbf{9 7 . 8 6}(0.75)$ & $88.64(1.18)$ & $93.76(0.89)$ & $94.57(1.14)$ \\
\hline UPSAMPLE & $96.07(1.54)$ & $96.09(1.2)$ & $96.6(1.06)$ & $93.5(1.14)$ & $\mathbf{9 7 . 8 7}(0.69)$ & $88.73(1.23)$ & $93.8(0.92)$ & $94.67(1.11)$ \\
PERTURB & $96.23(1.6)$ & $96.17(1.23)$ & $96.63(1.13)$ & $93.49(1.03)$ & $97.84(0.72)$ & $88.6(1.3)$ & $93.79(0.98)$ & $94.68(1.14)$ \\
512 & CVAE & $96.14(1.46)$ & $96.24(1.18)$ & $96.63(1.06)$ & $93.6(1.08)$ & $97.87(0.75)$ & $88.76(1.29)$ & $93.87(0.98)$ & $94.73(1.11)$ \\
& LINEAR & $96.07(1.54)$ & $96.11(1.21)$ & $96.6(1.06)$ & $93.49(1.13)$ & $\mathbf{9 7 . 8 7}(0.69)$ & $88.76(1.25)$ & $93.8(0.92)$ & $94.67(1.11)$ \\
& EXTRA & $96.07(1.54)$ & $96.13(1.18)$ & $96.6(1.06)$ & $93.5(1.14)$ & $\mathbf{9 7 . 8 7}(0.69)$ & $88.73(1.25)$ & $93.8(0.92)$ & $94.67(1.11)$ \\
DELTAR & $\mathbf{9 6 . 2 9}(1.52)$ & $\mathbf{9 6 . 2 9}(1.34)$ & $\mathbf{9 6 . 7 1}(1.1)$ & $\mathbf{9 3 . 8 7}(1.04)$ & $97.86(0.75)$ & $\mathbf{8 9 . 1 1}(1.22)$ & $\mathbf{9 4 . 0 3}(0.89)$ & $\mathbf{9 4 . 8 8}(1.12)$ \\
DELTAS & $96.19(1.61)$ & $96.2(1.23)$ & $96.69(1.07)$ & $93.61(0.96)$ & $97.86(0.75)$ & $88.84(1.28)$ & $93.83(0.94)$ & $94.74(1.12)$ \\
\hline
\end{tabular}

Table 7: IC accuracy on SNIPS dataset in the FSI setting, reported as mean (SD). The 10 seed examples are upsampled to 100 to train the feature extractor. 